\title{
Oi! - Opportunistic Data Transmission based on Wi-Fi Direct
}

\author{
Luis Lopes, Rute Sofia, Paulo Mendes, Waldir Moreira \\ COPELABS, University Lusofona \\ \{luis.amaral, rute.sofia, paulo.mendes, waldir.junior\}@ulusofona.pt
}

\begin{abstract}
This demo presents Oi!, an opportunistic data transmission tool. Implemented on Android and available as opensource, Oi! relies on social interaction, device-to-device shortrange wireless transmission, as well as on history of contact duration to perform opportunistic data transmission. In this demo we show how Oi! can be used with intermittent Internet access between sources and destinations, to transmit data in a reliable and yet opportunistic way.
\end{abstract}

\section{INTRODUCTION}

Opportunistic networks incorporate mobile devices that have a significant storage and battery capability, being also equipped with multiple short-range wireless interfaces. These devices can today communicate directly, not necessarily relying on an infrastructure-mode transmission. Moreover, these devices are carried by people and as such, they are often close and in a sufficient range to allow data transmission. The physical proximity of such devices is an aspect that is being integrated into aspects such as caching, or even routing. Nevertheless, from an operational perspective networking management and operational mechanisms do not take into consideration the social interaction of its human carriers. Hence, opportunistic networking today still requires a local wireless infrastructure, or Internet backbone access to allow data exchange among users that are often close physically.

Oi! ${ }^{1}[1]$ is an open-source software solution that supports opportunistic data transmission by exploiting the social interaction of people carrying personal devices that are wireless enabled. Oi! relies on device-to-device communication based on Wi-Fi Direct to assist in such transmission in a seamless and truly opportunistic way.

This demo explains how Oi! works in the context of intermittent connectivity. Section 2 describes the Oi! software architecture, while section 3 explains the demo.

\section{OI! ARCHITECTURE}

Oi! has been designed based on a modular approach in order to easily allow integration of novel opportunistic routing algorithms, as well as to integrate future naming schemes. The modular architecture of Oi! is illustrated in Fig. 1.

The User Interface (UI) module is used to compose messages (text, send photos, audio and video) to be sent to a recipient, based on the usual list of contacts. The UI is also used to see received messages. Each contact is associated with a specific MAC address (Wi-Fi Direct MAC address).

\footnotetext{
${ }^{1}$ Oi! means "Hello!" in Portuguese.
}

The Content Manager (CM) module is responsible to manage all of the messages to be sent, as well as received messages. Currently the CM stores messages in an XML format under specific directories. Both these modules reside at the OSI stack Application Layer level (Layer 7).

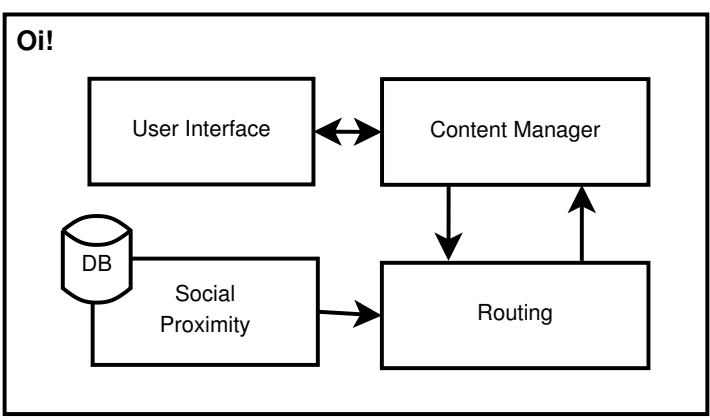

Fig. 1. Oi! architecture.

The Routing $(R T)$ module is provided separately to allow relying on any opportunistic forwarding mechanism. The current available forwarding mechanism is based on the dLife algorithm [2]. dLife relies on the history of contact duration to weigh the level of social interaction among users. By using social weight and node importance, dLife is able to perform less costly forwarding. Forwarding decisions are based on the level of social interaction (average contact durations over time) among devices: message replication only takes place when the social weight of an encountered node towards a specific destination is higher than the social weight of the current carrier of the message towards such destination.

The Social Proximity (SP) module [3] is responsible for gathering information about neighbor devices (via Bluetooth) ${ }^{2}$. It is also responsible for the social weight computation, information which it stores on a local SQLite database. Such database is used by the RT module whenever it needs to understand which next hop to select.

Once a successor for the message has been selected by the RT module, then the process of communication via $\mathrm{Wi}-\mathrm{Fi}$ Direct is established: Oi! triggers the setup of an Wi-Fi Direct communication, and opens a socket for the exchange of the message(s) to be sent to that node.

\footnotetext{
${ }^{2}$ We are currently working on a version that shall take into consideration peers obtained via Wi-Fi Direct, in order to allow to get peers within a larger range than Bluetooth.
} 


\section{A. Message Sending}

When a user wants to send a message, it writes one via the Oi! UI, picking a contact from the available contacts' list. This list carries a set of contacts (regular Smartphone Contact List) associated with a MAC address. When the user clicks the "Send" button, the message is stored (CM) in a specific directory, XML format. Periodically, the RT module checks all messages to be sent, by comparing available neighbors, their social weight towards the destination of each message. If a potential carrier (or the destination) is found, the RT triggers the creation of a socket (via Wi-Fi Direct) to the selected node.

\section{B. Message Reception}

Once a message is received by a device, it is stored in local memory (XML format), and in case that message is for the device, then it is sent to the UI for visualization. If the device is, however, an intermediate node, then the data is stored in the local directory for that purpose (internal memory) and the forwarding steps are periodically processed as previously explained.

\section{Demo Scenario}

Fig. 2 illustrates the demo, which comprises four Samsung Galaxy S3 smartphones, A, B, C, D running Android 4.2 (Jelly Bean). All of the devices have Oi! installed.

We start by having A isolated (cf. Fig. 2a), and none of the devices has Internet access. A wants to send a message to D. So it writes a message and pushes the send button. As there is no Internet access, this message is stored locally.
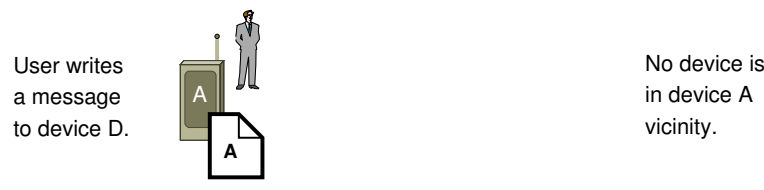

(a) User A aims to send message to User D who is not within Wi-Fi range.

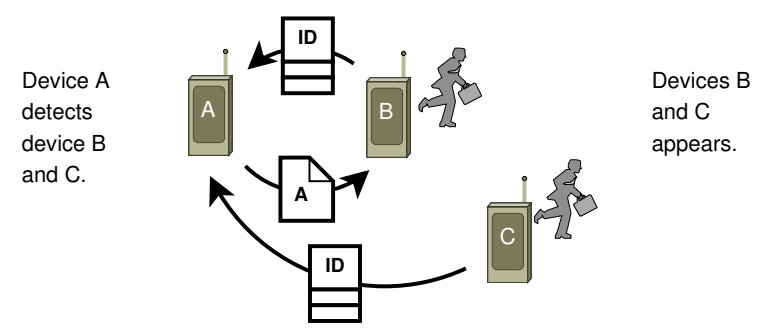

(b) Device A replicates message to a device with high probability to meet device D.

Devices $\mathrm{A}$ and $\mathrm{C}$ disappears.

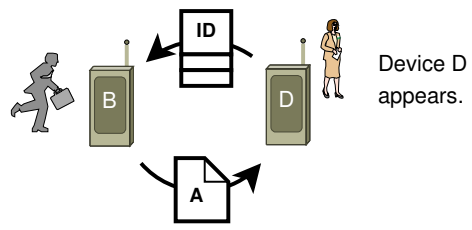

(c) Device B forward message to final destination, device D.

Fig. 2. Oi! Demonstration Scenario

A is walking around and meets devices B and C (cf., Fig. 2b). Oi! periodic Wi-Fi Direct scan detects that B and C are in the range. This process allows $\mathrm{A}$ to gather information via Wi-Fi Direct about B and C, namely: their identification, and a list of neighbors of $\mathrm{B}$ and $\mathrm{C}$ associated to the social weight of each node towards their neighbors.

The RT module in A checks the received list of peers holding their social weights towards thirds. Both devices B and $\mathrm{C}$ have met $\mathrm{D}$ in the past. However, as B meets D more frequently, it holds a higher social weight towards D than C. Hence, A selects B for potential successor to the message to be sent to D. A socket (via Wi-Fi Direct) is then established between $\mathrm{A}$ and $\mathrm{B}$ for the message to be exchanged.

Upon message reception, B detects that this message is not for itself, and so saves the message to be later forwarded to others devices that may carry the message to $\mathrm{D}$, or to be sent directly to $\mathrm{D}$.

Device B then meets D (cf., Fig. 2c), receiving its identification and respective list of social weights. This triggers a check of the messages to be sent, and the message from A to $\mathrm{D}$ is directly sent from $\mathrm{B}$ to $\mathrm{D}$.

\section{SUMMARY}

This demo concerns Oi!, an opportunistic application that considers social interaction as the basis for opportunistic forwarding. Available under LGPLv3.0, Oi! supports intermittent connectivity and takes into consideration history about prior contacts between devices to improve delivery reliability while at the same time reducing the overhead derived from keeping state concerning messages in multiple devices.

A new version fully based in Wi-Fi Direct (where additional meta-dataand peers are also obtained via Wi-Fi direct) is being developed. Moreover, Oi! is being validated in the context of the Named-Data Networking worldwide testbed.

As future work, we plan to carry out large-scale tests to assess the scalability and performance of the application in a real-world setting (e.g., city).

\section{ACKNOWLEDGMENT}

The research leading to these results has received funding from the European Union (EU) Horizon 2020 research and innovation programme under grant agreement No 645124 (Action full title: Universal, mobile-centric and opportunistic communications architecture, Action Acronym: UMOBILE). This paper reflects only the authors views and the Community is not liable for any use that may be made of the information contained therein.

\section{REFERENCES}

[1] L. Lopes, R. C. Sofia, P. Mendes, and W. Moreira, "Oi!: An opportunistic data transmission tool based on social-aware routing, version 2.0." COPE-SW-16-01, January 2016. Available at http://copelabs.ulusofona.pt/scicommons/index.php/publications/show/853.

[2] W. Moreira, P. Mendes, and S. Sargento, "Opportunistic routing based on daily routines," in Proc. of WoWMoM, pp. 1-6, June 2012.

$\begin{array}{llll}\text { [3] W. Moreira, } & \text { "Social } & \text { proximity } & \text { library." } \\ \text { COPE-SW-15-02, } & \text { Sept. } & 2015 . & \text { Available }\end{array}$ http://copelabs.ulusofona.pt/scicommons/index.php/publications/show/801. 